\title{
Insulin-induced gene 1 and 2 isoforms synergistically regulate triacylglycerol accumulation, lipid droplet formation, and lipogenic gene expression in goat mammary epithelial cells
}

\author{
Cong Li, ${ }^{1}$ Miao Wang, ${ }^{1}$ Tianying Zhang, ${ }^{1}$ Qiuya He, ${ }^{1}$ Huaiping Shi, ${ }^{1}$ Jun Luo, ${ }^{1 *}$ and Juan J. Loor ${ }^{2 *}$ \\ ${ }^{1}$ Shaanxi Key Laboratory of Molecular Biology for Agriculture, College of Animal Science and Technology, Northwest A\&F University, Yangling, \\ Shaanxi 712100, PR China \\ ${ }^{2}$ Mammalian NutriPhysioGenomics, Department of Animal Sciences and Division of Nutritional Sciences, University of Illinois, Urbana 61801
}

\section{ABSTRACT}

The insulin-induced genes INSIG1 and INSIG2 (INSIG) are known to regulate adipogenesis in nonruminants. Although data in bovine mammary tissue underscore a role for INSIG1 during lactation, regulatory mechanisms of INSIG action in ruminant mammary lipid metabolism are not well known. In the present study, INSIG1 and INSIG2 were overexpressed or silenced through adenoviral transfection to evaluate their role in lipid metabolism in goat mammary epithelial cells (GMEC). The INSIG were overexpressed using an adenovirus system with recombinant green fluorescent protein as the control. Downregulation of INSIG was performed via small interfering RNA targeting INSIG with a scrambled small interfering RNA as a negative control. The GMEC were treated with these constructs for $48 \mathrm{~h}$ before analyses. Responses to overexpressing INSIG1 or INSIG2 included downregulation of SREBF1, ACACA, FASN, SCD1, GPAM, DGAT2, $A T G L$, and $H S L$ coupled with a decrease in content of triacylglycerol (TAG), total cholesterol (TC), and lipid droplet accumulation. The marked decrease in content of TAG and TC in response to overexpression of $I N$ SIG2, along with a modest decrease in content of TAG when INSIG1 was overexpressed, suggested that TAG synthesis is mainly regulated by INSIG2, whereas TC synthesis is equally regulated by INSIG2 and INSIG1. The lack of difference in mRNA expression of genes related to lipid metabolism, content of TAG, and accumulation of lipids in response to interference alone of INSIG1 or INSIG2 indicated that INSIG proteins play a biological role in the maintenance of lipid homeostasis. However, in response to simultaneous interference of INSIG1 and INSIG2, the marked increase in content

\footnotetext{
Received August 2, 2018.

Accepted October 30, 2018

*Corresponding authors: luojun@nwafu.edu.cn and jloor@illinois
} .edu of TAG and TC and accumulation of lipids along with significant upregulation of SREBF1, ACACA, SCD1, AGPAT6, and DGAT2 suggested that INSIG1 and INSIG2 synergistically regulate milk fat synthesis in GMEC. These results highlight an essential role of INSIG in regulating lipid synthesis in dairy goat mammary cells and underscore the complexity of mammary lipid synthesis in ruminants.

Key words: insulin-induced gene, lipid metabolism, RNA interference, overexpression

\section{INTRODUCTION}

In nonruminants, the insulin-induced genes INSIG1 and INSIG2 (INSIG), located in the outer endoplasmic reticulum membrane, play an essential role in regulation of lipid metabolism (Yabe et al., 2003; Bionaz et al., 2012). Previous studies (Dong and Tang, 2010; Smith et al., 2010) in humans on the expression and distribution of INSIG1 and INSIG2 revealed that INSIG1 has the highest mRNA expression in liver, accounting for more than $75 \%$ of the total INSIG. In contrast, INSIG2 is widely expressed in most tissues, and its expression accounts for half of INSIG1 (Krapivner et al., 2007). In dairy cows, INSIG1 mRNA in bovine mammary tissue at peak lactation is about 14 times higher than in the dry period (Bionaz and Loor, 2008). Differentiation of murine preadipocytes is associated with a 12 -fold upregulation of INSIG2 mRNA (Krapivner et al., 2008). Regulation of INSIG1 and INSIG2 differs, underscoring potential differences in terms of their role in lipid metabolism. For instance, INSIG1 plays a central role in cholesterol homeostasis in rodent liver (Gong et al., 2006), whereas INSIG2 controls differentiation of precursor adipocytes (Lee and DeBose-Boyd, 2010). Therefore, available evidence underscores the potential importance of INSIG genes in aspects related to lipid metabolism.

Most of the previous published studies on the relationship between INSIG and lipid metabolism in 
humans and mice have focused on the liver and fat cells (Li et al., 2003; Cheng et al., 2015; Wang et al., 2016; Lee et al., 2017; Linden et al., 2018). Whether these genes play a similar role in goat mammary tissue remains unclear. The hypothesis was that INSIG1 and INSIG2 encode proteins with critical roles in the regulation of lipid metabolism in goat mammary epithelial cells (GMEC). Therefore, the objective was to evaluate the function of INSIG in lipid metabolism of GMEC. We measured expression levels of INSIG in dairy goat mammary tissue at different stages of lactation and used adenovirus-mediated overexpression and RNA interference approaches to uncover their role in triacylglycerol (TAG) and cholesterol metabolism.

\section{MATERIALS AND METHODS}

\section{Ethics Statement}

The Animal Care and Use Committee (permit number 15516) of Northwest A\&F University (Yangling, Shaanxi, China) approved all procedures involved in the use of live goats.

\section{Goat Mammary Tissue Collection}

Twelve multiparous and mastitis-free Xinong Saanen dairy goats from the experimental farm at Northwest A\&F University were selected for the study. Mammary gland tissues from goats $(\mathrm{n}=3$ biological replicates from each lactation stage) in early lactation ( $15 \mathrm{~d}$ postpartum), peak lactation (60 d postpartum), mid lactation (120 d postpartum), and the nonlactating period (60 d before parturition) were harvested via biopsy. Percutaneous biopsies were performed from the right udder according to methods described previously (Farr et al., 1996). Briefly, after making a skin incision, blunt dissection of the mammary capsule was performed to ensure that the tissue obtained was mammary parenchyma during the biopsy. All mammary samples were obtained under sterile conditions and immediately frozen in liquid nitrogen until RNA extraction.

\section{Adenovirus Generation and Overexpression of INSIG1 and INSIG2}

Generation and proliferation of the adenoviruses were performed as previously described (Luo et al., 2007; Shi et al., 2017). Briefly, the cDNA sequences of dairy goat INSIG1 (GenBank no. NM_001286088.1) and INSIG2 (GenBank no. NM_001285738.1) were subcloned into the pAdTrack-CMV shuttle plasmid vector between the SalI and BglI (New England BioLabs Inc., Ipswich, MA) restriction sites to generate pAdTrack-CMV-INSIG1 and pAdTrack-CMV-INSIG2 vectors. Subsequently, these 2 vectors were inserted into an adenoviral vector (pAdEasy-1) to generate adenoviral plasmids in BJ5183 bacterial cells. The adenoviral plasmids linearized by PacI (New England BioLabs Inc.) were transfected into 293A cells to generate the adenovirus pAd-INSIG1 and pAd-INSIG2. The recombinant adenovirus with green fluorescent protein (Ad-GFP) was used as a negative control.

\section{RNA Interference}

To knock down expression of INSIG1 and INSIG2, small interfering RNA (siRNA) were designed using online software (http://sirna.wi.mit.edu/home.php), and synthesized by Invitrogen Corp. (Carlsbad, CA). Two siRNA [small interfering RNA target INSIG1 (siINSIG1)-100, siINSIG1-547; small interfering RNA target INSIG2 (siINSIG2)-175, siINSIG2-244] for each gene were designed to obtain an ideal transfection efficiency (Supplemental Figure S1; https://doi.org/10 .3168/jds.2018-15492). A scrambled siRNA (siNC) was designed and used as a negative control. The siRNA sequences are listed in Supplemental Table S1 (https:/ /doi.org/10.3168/jds.2018-15492).

For INSIG mRNA interference, cells cultured in 6-well plates were transfected with $50 \mathrm{n} M$ siRNA using transfection reagent (Lipofectamine RNAiMAX, Thermo Fisher Scientific Inc., Waltham, MA) in lactogenic medium without antibiotic. The transfection was performed according to the manufacturer's instructions. Transfected GMEC were collected after $24 \mathrm{~h}$ of initial culture and then harvested at $48 \mathrm{~h}$ ( $24 \mathrm{~h}$ later) for RNA extraction and subsequent assays.

\section{Cell Culture and Treatment}

The GMEC were isolated from Xinong Saanen dairy goats at peak lactation as previously described (Wang et al., 2010; Shi et al., 2014). Details of cell culture were also described in our previous studies (Lin et al., 2013; Shi et al., 2013). Briefly, cells were incubated at $37^{\circ} \mathrm{C}$ in $5 \% \mathrm{CO}_{2}$. Culture medium comprised Dulbecco's modified Eagle medium/F12 (Hyclone, Beijing, China) containing insulin ( $5 \mathrm{mg} / \mathrm{L}$; Sigma-Aldrich, St. Louis, MO), hydrocortisone (5 mg/L; Sigma-Aldrich), penicillin/streptomycin (10,000 units/L; Harbin Pharmaceutical Group, Harbin, China), epidermal growth factor (1 mg/L; Sigma-Aldrich), and fetal bovine serum (10\%; Gibco, Waltham, MA). To promote lactogenesis, GMEC were cultured in medium described above plus prolactin $(2 \mu \mathrm{g} / \mathrm{mL}$; Sigma-Aldrich $)$ for $24 \mathrm{~h}$ before initial experiments. The transfected 293A cells were cultured in the basal DMEM medium (Gibco) contain- 
ing $10 \%$ fetal bovine serum. The GMEC at about $80 \%$ confluence were transfected with adenovirus supernatant [recombinant adenovirus expressing INSIG1 (AdINSIG1), recombinant adenovirus expressing INSIG2 (Ad-INSIG2), and Ad-GFP). The transfected GMEC were collected after $48 \mathrm{~h}$ of culture for lipid extraction, total RNA extraction, and TAG synthesis.

\section{RNA Extraction and Quantitative Real-Time PCR}

Total RNA was extracted from goat mammary tissue via the Trizol method (Invitrogen Corp., Carlsbad, CA) according to the manufacturer's instructions. Total RNA from GMEC was extracted using the RNA Prep Pure cell kit (Tiangen Biotech Co. Ltd., Beijing, China) according to the manufacturer's protocol. The RNA was treated with DNase (Tiangen Biotech Co. Ltd.) to remove genomic DNA contamination. The RNA quality was examined by agarose gel electrophoresis analysis of $28 \mathrm{~S}$ and $18 \mathrm{~S}$ rRNA. Synthesis of cDNA was conducted using the PrimeScript RT kit (Takara Bio Inc., Ostu, Japan) according to the manufacturer's directions. Quantitative real-time PCR was performed according to the manufacturer's instructions using SYBR Green (SYBR Premix Ex TaqII, Perfect Real Time, Takara Bio Inc., Dalian, China) on a CFX96 Real-Time PCR Detection System (Bio-Rad, Hercules, $\mathrm{CA}$ ). The PCR amplification was carried out at $95^{\circ} \mathrm{C}$ for $4 \mathrm{~min}$ followed by 40 cycles at $95^{\circ} \mathrm{C}$ for $15 \mathrm{~s}, 60^{\circ} \mathrm{C}$ for $30 \mathrm{~s}$, and $72^{\circ} \mathrm{C}$ for $30 \mathrm{~s}$. The presence of a single PCR product was confirmed by the dissociation protocol using incremental temperatures to $95^{\circ} \mathrm{C}$ for $15 \mathrm{~s}$ and $65^{\circ} \mathrm{C}$ for $5 \mathrm{~s}$.

Genes evaluated include those related to fatty acid (FA) de novo synthesis and desaturation [fatty acid synthase $(F A S N)$, acetyl-CoA carboxylase $\alpha(A C A C A)$, and stearoyl-CoA desaturase ( $S C D 1)$ ], transcription factors [cleavage activating protein $(S C A P)$ and sterol regulatory element-binding transcription factor 1 (SREBF1)], TAG synthesis [glycerol-3-phosphate acyltransferase (GPAM), 1-acylglycerol-3-phosphate O-acyltransferase 6 (AGPAT6), and diacylglycerol O-acyltransferase 2 (DGAT2)] and TAG hydrolysis [adipose triglyceride lipase $(A T G L)$ and hormone sensitive lipase $(H S L)]$. The gene expression values were calculated using the relative quantification $\left(2^{-\Delta \Delta \mathrm{Ct}}\right)$ method (Oppi-Williams et al., 2013); ubiquitously expressed transcript (UXT), mitochondrial ribosomal protein L39 (MRPL39), and ribosomal protein $\mathrm{S} 9(R S P 9)$ were used as endogenous references for normalization of targeted RNA profiles, and 3 technical replicates were analyzed per sample (Bionaz and Loor, 2007; Kadegowda et al., 2009; Bonnet et al., 2013). The primer sequences of the above genes are included in Supplemental Table S2 (https:/ / doi.org/10.3168/jds.2018-15492).

\section{Cellular TAG and Total Cholesterol Assay}

Concentration of TAG was measured with a commercial kit (Applygen Technologies Inc., Beijing, China) according to the manufacturer's instructions. Briefly, adenovirus-infected or siRNA-transfected cells were washed 3 times with PBS and harvested in lysis buffer (50 $\mathrm{m} M$ Tris-HCL, pH 7.4, $150 \mathrm{~m} \mathrm{M} \mathrm{NaCl,} \mathrm{1 \%} \mathrm{Triton}$ X-100; Beijing Solarbio Science \& Technology Co., Ltd, Beijing, China), and cell lysates then extracted. The TAG and total cholesterol (TC) contents were measured by enzymatic colorimetry using a commercial kit (GPO Trinder reaction, Applygen Technologies Inc.). The BCA protein kit (Pierce, Thermo Fisher Scientific Inc.) was used to determine the total protein concentration. The contents of TAG and TC were determined by normalization to the total protein of each sample, and data were reported as milligrams per gram of protein.

\section{Oil Red O Staining}

The GMEC cultured in 6-well plates (Nunc, Roskilde, Denmark) were incubated with adenovirus supernatant for $48 \mathrm{~h}$ and then washed with PBS and fixed in $10 \%$ (vol/vol) paraformaldehyde for $1 \mathrm{~h}$ at $4^{\circ} \mathrm{C}$. After rinsing again with PBS, cells were stained with $5 \%$ Oil Red O in isopropanol for $30 \mathrm{~min}$ at room temperature and then rinsed repeatedly to remove impurities. A system incorporated in a microscope (Leica, Wetzlar, Germany) was applied to capture representative photomicrographs at $200 \times$ magnification, and pictures were taken according to a published procedure (Mehlem et al., 2013).

\section{Statistical Analysis}

Data for all measurements are from triplicate incubations, and all the experimental data are reported as means \pm standard error of the mean. An ANOVA (SAS Institute Inc., Cary, NC) was used for statistical comparisons and for determining the differences of mRNA expression of genes and total TAG or TC among treatments. The statistical model was

$$
x_{i j}=\mu+\alpha_{i}+\varepsilon_{i j},
$$

where $x_{i j}$ is the experimental variable, $\mu$ is the overall mean response, $\alpha_{i}$ is the effect of the $i$ th level $\mathrm{A}_{i}$ of factor $\mathrm{A}$, and $\varepsilon_{i j}$ is the random residual. For instance, if $x_{i j}$ refers to relative mRNA expression of INSIG1 or INSIG2, $\alpha_{i}$ pertains to the effect of 4 different lacta- 
tion stages; if $x_{i j}$ refers to mRNA expression of genes, $\alpha_{i}$ pertains to the treatment effect of control and overexpression or interference of INSIG1/INSIG2; and if $x_{i j}$ refers to cellular TAG and TC content, $\alpha_{i}$ pertains to the treatment effect of control and overexpression or interference of INSIG1/INSIG2. The statistical significance of differences was evaluated with either Student's 2 -tailed $t$-test or the 1-way ANOVA when appropriate. Differences were considered statistically significant at $P<0.05$.

\section{RESULTS}

Transcript Abundance of INSIG Isoforms in Mammary Tissue of Dairy Goats at Different Stages of Lactation

As shown in Figure 1, the expression level of INSIG1 was higher than that of INSIG2. A significant upregulation of INSIG1 mRNA was detected in early, peak, and mid lactation compared with the dry period $(P<0.05)$. No differences were detected among lactation periods (Figure 1A). Relative to mid lactation, the expression level of INSIG2 in early and peak lactation was dramatically increased $(P<0.05)$, whereas no significant change was observed relative to the dry period (Figure

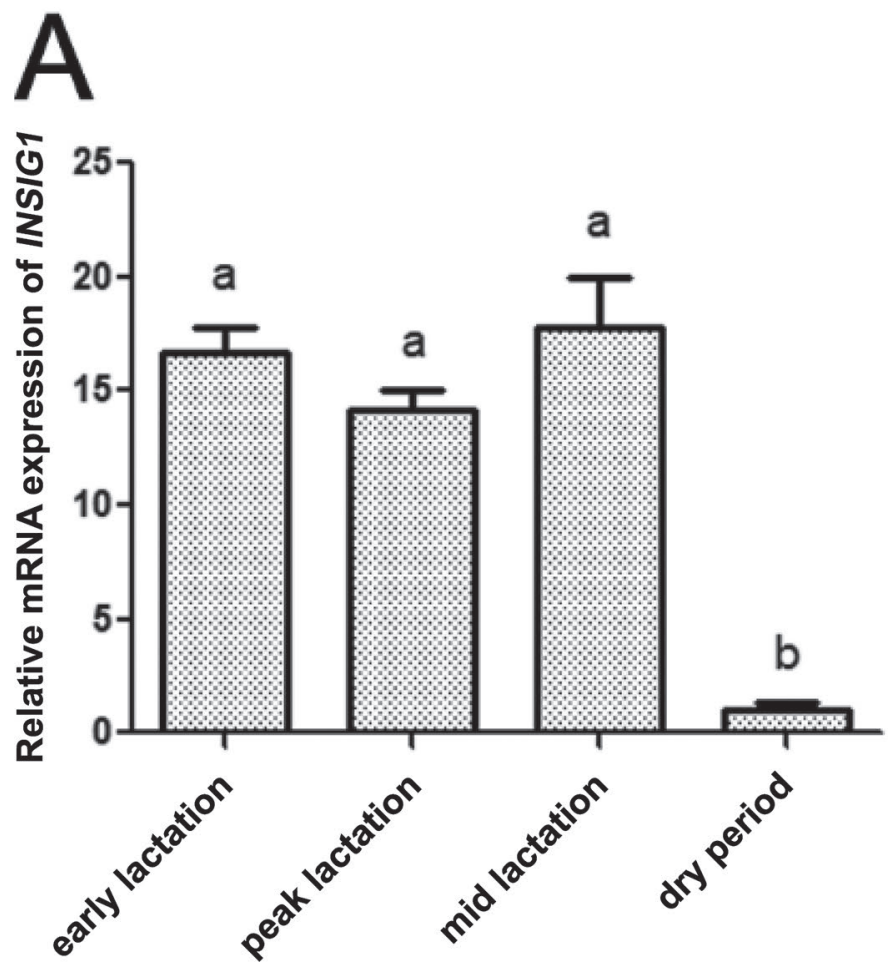

1B). During the whole lactation, INSIG1 and INSIG2 mRNA reached the highest expression at mid and peak lactation, respectively (Figure 1A and B).

\section{Overexpression of INSIG Suppresses mRNA Expression of Key Genes Related to Lipogenesis}

As shown in Figures 2 and 3, mRNA expression of INSIG1 in the cultures with Ad-INSIG1 was almost 500 -fold $(P<0.01)$ greater than that of Ad-GFP (Figure 2A), and expression of INSIG2 was greater $(P<0.01)$ in cultures with Ad-INSIG2 (Figure 3A). Compared with the Ad-GFP control, no significant effect on the expression of $S C A P$ and SREBF1 in cells infected with Ad-INSIG1 was observed (Figure 2B). Overexpression of INSIG2 had no effect on SCAP but had a tendency to reduce the expression of $S R E B F 1$ and markedly suppressed mRNA abundance of INSIG1 $(P<0.05$; Figure 3B). Compared with the Ad-GFP control, the overexpression of INSIG1 markedly downregulated the expression of $A C A C A, F A S N$, and $S C D 1$ $(P<0.05$; Figure $2 \mathrm{C})$. A tendency for $F A S N$ downregulation was observed when INSIG2 was overexpressed, whereas expression of $A C A C A$ and $S C D 1$ was markedly downregulated $(P<0.05)$ compared with the negative control (Figure $3 \mathrm{C}$ ).

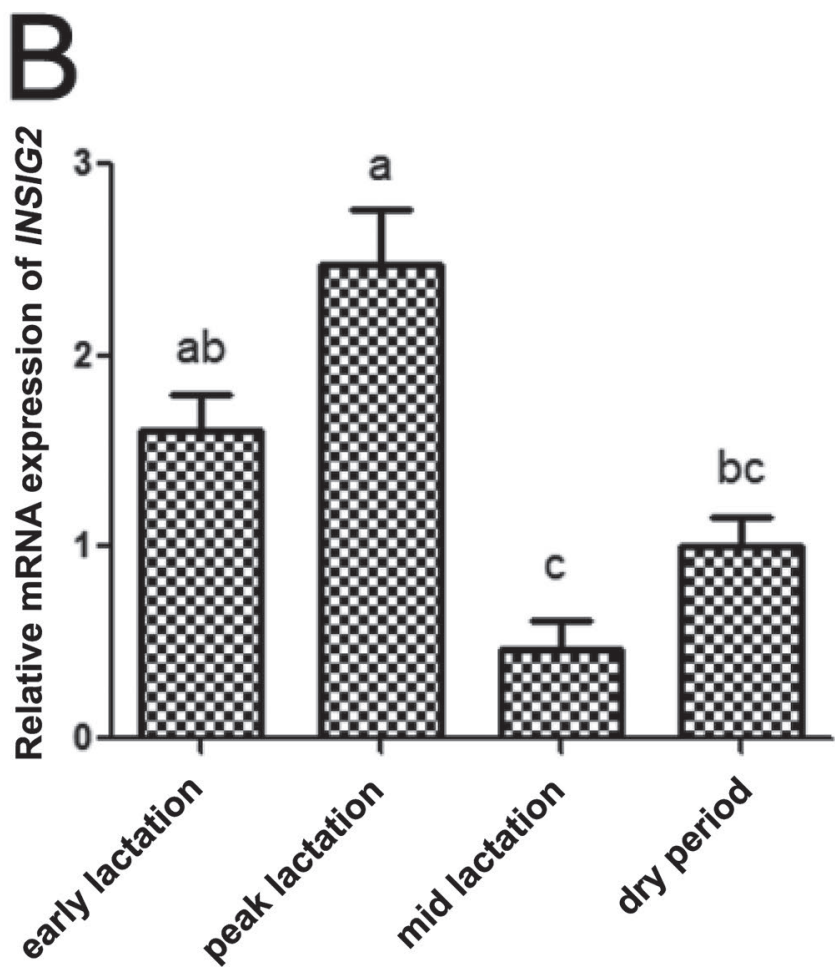

Figure 1. Relative mRNA expression of (A) INSIG1 and (B) INSIG2 in goat mammary tissues at different lactation stages. Samples were collected in 4 lactation stages (early lactation, peak lactation, mid lactation, and dry period). Quantitative real-time PCR was applied and data were analyzed using the relative quantification $\left(2^{-\Delta \Delta \mathrm{Ct}}\right)$ method. Values are presented as means $\pm \mathrm{SE}$. The different letters $(\mathrm{a}-\mathrm{c})$ represent significant differences $(P<0.05)$ in gene expression. 

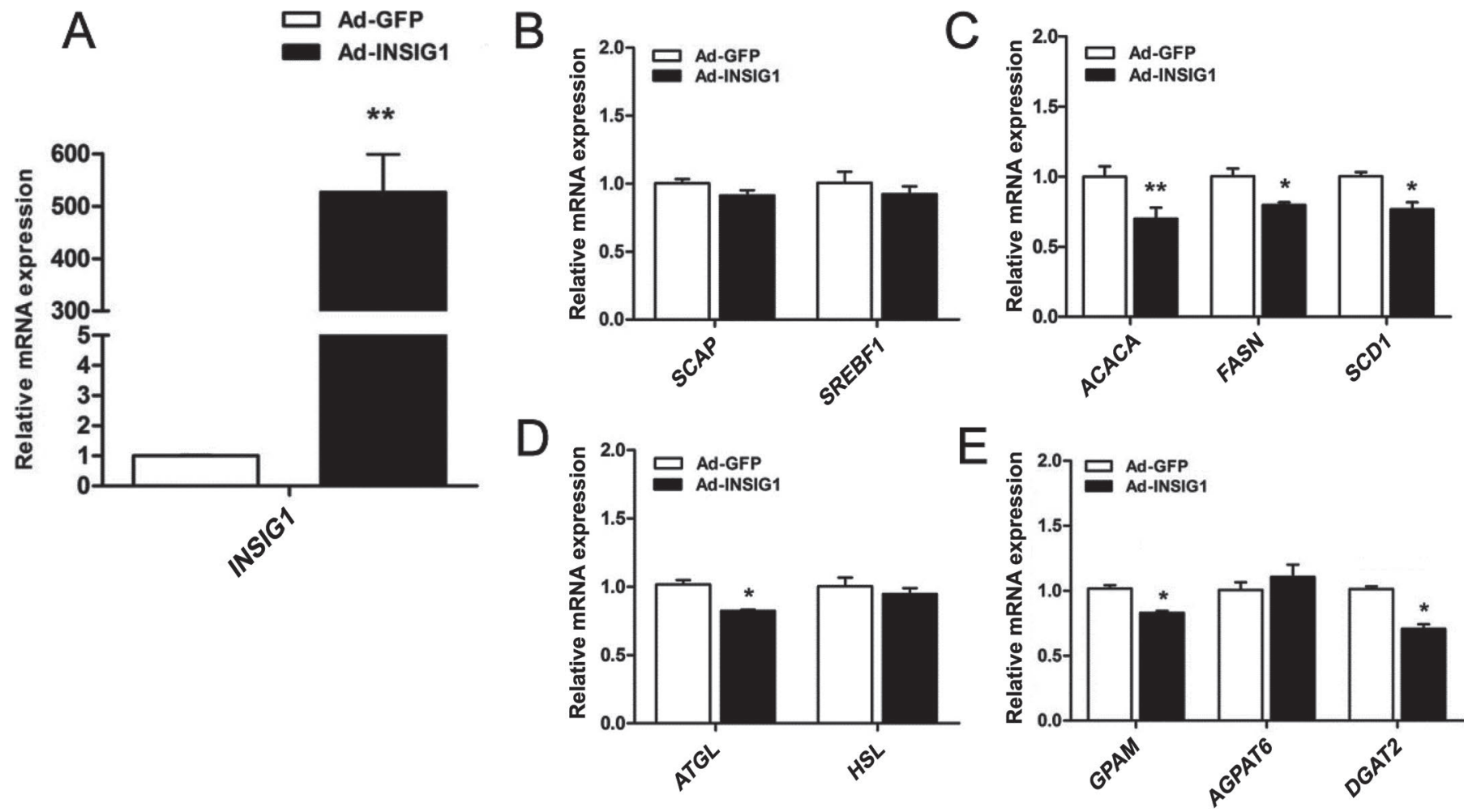

Figure 2. Overexpression of INSIG1 suppressed mRNA expression of genes related to lipid synthesis in goat mammary epithelial cells (GMEC). The GMEC were transfected with a recombinant adenovirus expressing INSIG1 (Ad-INSIG1) or the control (adenovirus containing green fluorescent protein; Ad-GFP) for $48 \mathrm{~h}$ and then collected for mRNA extraction. (A) INSIG1 mRNA expression level. The mRNA expression levels of genes related to (B) transcription factors, (C) de novo fatty acid synthesis and desaturation, (D) triacylglycerol hydrolysis, and (E) triacylglycerol synthesis in GMEC. Values are presented as means \pm SE from 3 individual cultures. ${ }^{*} P<0.05$ and $* * P<0.01$ compared with control (Ad-GFP).

Compared with the Ad-GFP control, overexpression of INSIG1 did not affect the mRNA abundance of AGPAT6 but decreased the expression of GPAM and DGAT2 $(P<0.05$; Figure $2 \mathrm{E})$. Overexpressing INSIG2 markedly downregulated mRNA abundance of DGAT2 and AGPAT6 $(P<0.05)$, whereas no change in GPAM mRNA was observed (Figure 3E). Relative to the Ad-GFP control, the cells overexpressing INSIG1 had downregulation of ATGL $(P<0.05)$, but $H S L$ was not affected (Figure 2D). Treatment with Ad-INSIG2 markedly downregulated $H S L(P<0.05)$, and $A T G L$ mRNA tended to be downregulated relative to the Ad-GTP control (Figure 3D). Collectively, these data suggested that overexpression of INSIG1 and INSIG2 suppressed lipid accumulation and simultaneously inhibited lipolysis in GMEC.

\section{Overexpression of INSIG Affects TAG Accumulation, TC Content, and Lipid Droplet Formation}

Relative to the Ad-GFP control, lipid droplet accumulation was markedly decreased upon INSIG2 overexpression $(P<0.05)$ and Ad-INSIG1 treatment
(Figure 4A). Compared with the Ad-GFP control, the overexpression of INSIG2 markedly reduced total cellular TAG $(P<0.01$; Figure 4D). However, no significant change in TAG was detected in cells overexpressing $I N$ SIG1 compared with the control Ad-GFP (Figure 4B). Both Ad-INSIG1 and Ad-INSIG2 treatments markedly decreased TC content compared with the Ad-GFP infected cells $(P<0.05$; Figure $4 \mathrm{C}$ and $\mathrm{E})$.

\section{Simultaneous Interference of INSIG Enhances mRNA Expression of Key Genes Related to Lipogenesis}

Compared with the siNC control, siRNA for siINSIG1-547 and siINSIG2-175 effectively knocked down endogenous expression of INSIG1 and INSIG2 by an average of 80 and $85 \%$, respectively $(P<0.05$; Supplemental Figure S1, https://doi.org/10.3168/jds.2018 -15492). Thus, these 2 siRNA were used in the subsequent interference experiment. The quantitative PCR results (Figure 5A) indicated that incubation with siINSIG1 decreased abundance of INSIG1 and had no effect on the mRNA abundance of INSIG2. Compared with the siNC control, incubation with siINSIG2 de- 
creased INSIG2 and simultaneously reduced abundance of INSIG1 by approximately $28 \%$ (Figure $5 \mathrm{~A}$ ). In addition, compared with the siNC control, the GMEC treated with siINSIG1 and siINSIG2 together decreased the mRNA expression of INSIG1 and INSIG2 by more than $80 \%$. Therefore, as depicted in Figure 5A, the selected siRNA effectively knocked down endogenous INSIG1 and INSIG2 gene expression.

Relative to the siNC control, incubation with siINSIG1 or siINSIG2 alone had no significant effect on expression of genes associated with lipid metabolism, including SCAP, SREBF1, ACACA, FASN, SCD1, GPAM, AGPAT6, and DGAT2 (Figure 5B-D). However, compared with the siNC control, simultaneous incubation with siINSIG1 and siINSIG2 led to a marked upregulation of mRNA abundance of SREBF1, $A C A C A, S C D 1, A G P A T 6$, and DGAT2 $(P<0.05)$. Furthermore, there was a tendency for upregulation of $F A S N$ mRNA, whereas expression of GPAM was downregulated (Figure 5B-D).

\section{Interference of INSIG Increases TAG Accumulation, TC Content, and Lipid Droplet Formation}

Compared with the siNC control, incubation with siINSIG1 or siINSIG2 alone induced lipid droplet accumulation, whereas siINSIG1 and siINSIG2 together led to a marked increase in lipid droplet accumulation (Figure 6A). Compared with the siNC control (Figure $6 \mathrm{~B})$, knockdown of INSIG1 or INSIG2 alone had no significant effect on cellular TAG content, whereas interference of INSIG1 and INSIG2 together markedly increased $(P<0.05)$ TAG accumulation. Relative to the siNC control, the cellular TC content was enhanced regardless of interference of INSIG alone or in combination (Figure 6C).

\section{DISCUSSION}

The change in the profile of INSIG1 and INSIG2 in mammary tissue across stages of lactation agrees with

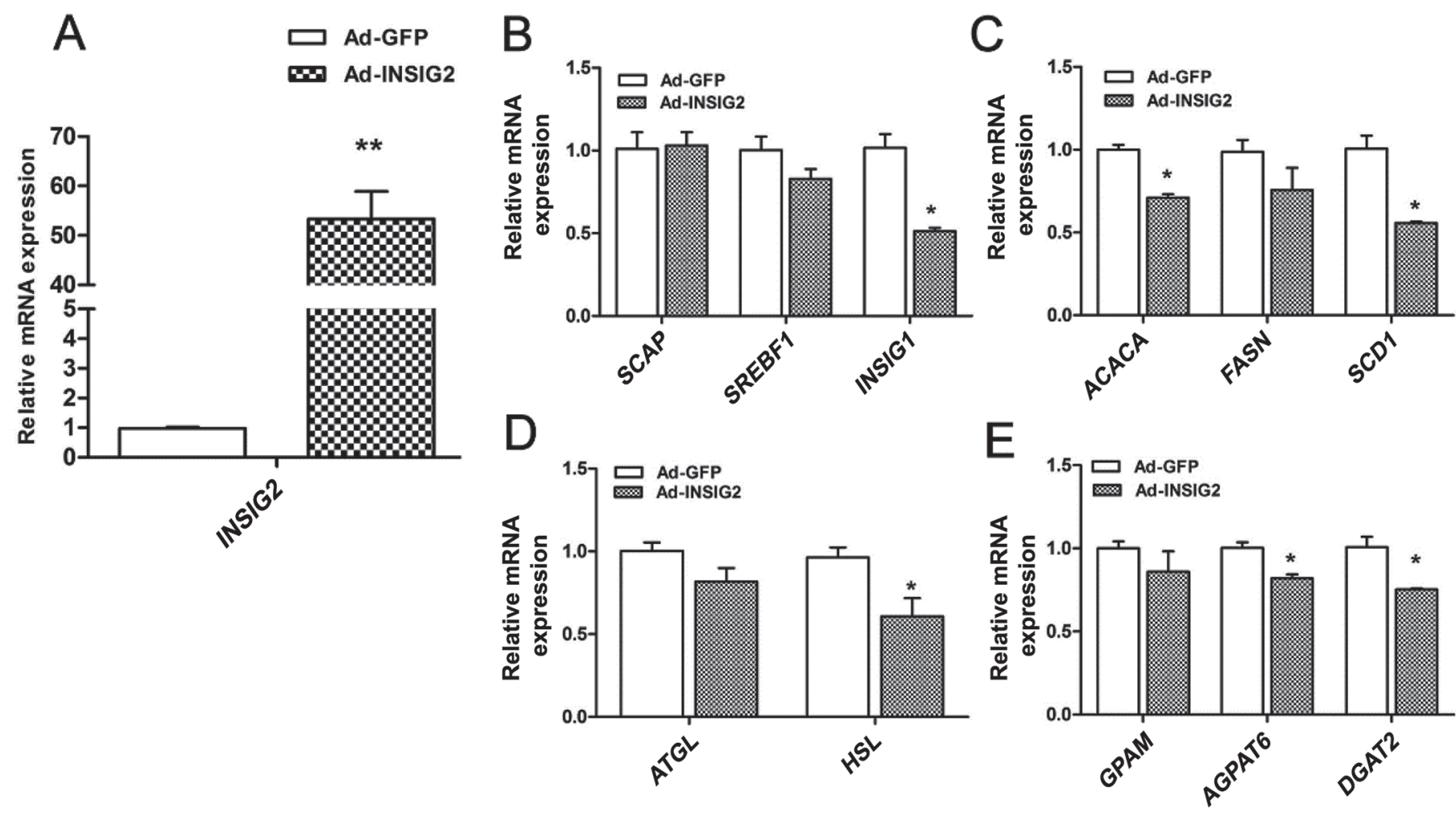

Figure 3. Overexpression of INSIG2 repressed mRNA expression of genes related to lipid synthesis in goat mammary epithelial cells (GMEC). The GMEC were transfected with a recombinant adenovirus expressing INSIG2 (Ad-INSIG2) or the control (adenovirus containing green fluorescent protein; Ad-GFP) for $48 \mathrm{~h}$ and then collected for mRNA extraction. (A) INSIG2 mRNA expression level. The mRNA expression levels of genes related to (B) transcription factors, (C) de novo fatty acid synthesis and desaturation, (D) triacylglycerol hydrolysis, and (E) triacylglycerol synthesis in GMEC. Values are presented as means \pm SE from 3 individual cultures. ${ }^{*} P<0.05$ and ${ }^{* *} P<0.01$ compared with control (Ad-GFP). 
the dynamics in goat milk fat composition and abundance of genes related to lipid metabolism (Romero et al., 2013). In addition, the much higher expression of INSIG1 mRNA at mid lactation and the higher expression of INSIG2 at peak lactation underscore the potentially different biological functions for these 2
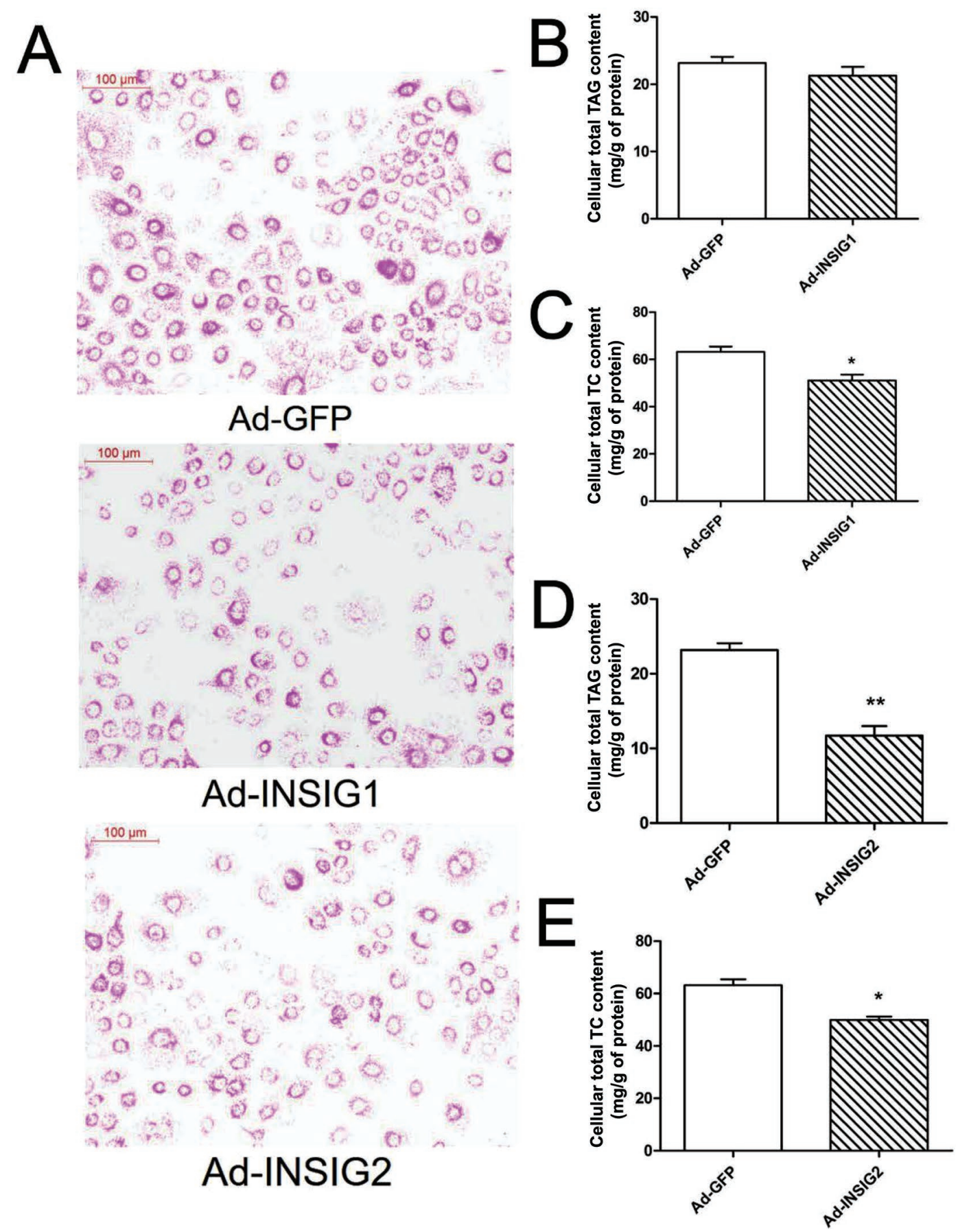

Figure 4. Overexpression of INSIG1 and INSIG2 decreased the accumulation of lipid droplet, concentration of cellular triacylglycerol (TAG), and total cholesterol (TC). The goat mammary epithelial cells (GMEC) were transfected with a recombinant adenovirus expressing INSIG1 (Ad-INSIG1), adenovirus expressing INSIG2 (Ad-INSIG2), and the control (adenovirus containing green fluorescent protein; Ad-GFP) and collected at $48 \mathrm{~h}$ for lipid droplet measurement and TAG and TC analysis. (A) Lipid droplet formation after Oil Red O treatment (scale bar: 100 $\mu \mathrm{m})$. (B) Total TAG content in GMEC incubated with Ad-INSIG1. (C) Total TC content in GMEC incubated with Ad-INSIG1. (D) Total TAG content in GMEC incubated with Ad-INSIG2. (E) Total TC content in GMEC incubated with Ad-INSIG2. Values are presented as means \pm SE from 3 individual cultures. The contents of TAG and TC are presented as milligrams per gram of protein. ${ }^{*} P<0.05$ and ${ }^{* *} P<0.01$ compared with control (Ad-GFP). 
isoforms during the lactation cycle. These results led us to hypothesize that INSIG plays a crucial role in mammary lipogenesis and TAG synthesis.

Work in rodent models has revealed that INSIG binds $S C A P$ and inhibits the activation of SREBF protein but has little effect on their mRNA level (Yang et al., 2002). The lack of change in mRNA expression of SCAP and SREBF1 when INSIG1 was overexpressed agrees with in vivo studies in mice (Engelking et al., 2004; Takaishi et al., 2004). Despite the lack of effect on SCAP mRNA when INSIG2 was overexpressed, the tendency for downregulation of SREBF1 mRNA along with the downregulation of INSIG1 mRNA suggested a role of INSIG2 in transcriptional regulation in GMEC.

The downregulation of $A C A C A, F A S N$, and $S C D 1$ (target genes of $S R E B F 1$ ) in response to overexpres- sion of INSIG1 agrees with previous studies in mice (Engelking et al., 2004; Takaishi et al., 2004). Thus, the present data suggest that activation of INSIG1 transcription inhibits the activation of SREBF protein along with the transcription of its target genes. An important function of INSIG1 appears to be the inhibition of de novo synthesis and desaturation of FA. The downregulation of $A C A C A$ and $S C D 1$ in response to INSIG2 overexpression also agrees with previous data in mice (Takaishi et al., 2004). Together, the data suggest that both INSIG1 and INSIG2 could affect de novo synthesis and desaturation of FA.

Milk fat TAG synthesis is partly controlled by 3 key enzymes: GPAM is involved in the first step reaction (rate-limiting step), AGPAT catalyzes the esterification of the $s n-2$ position of glycerol-3-phosphate, and DGAT
A

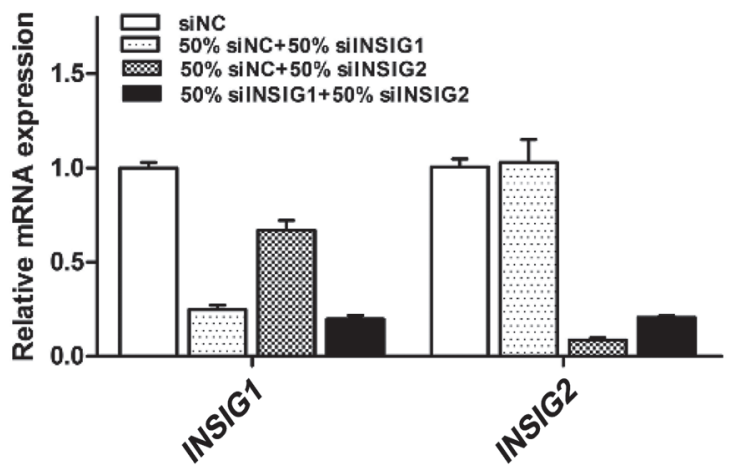

C

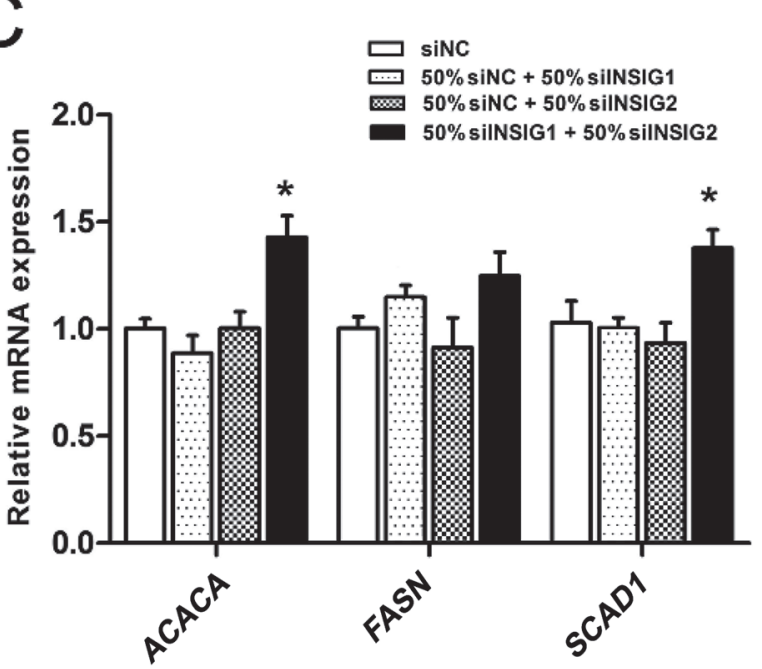

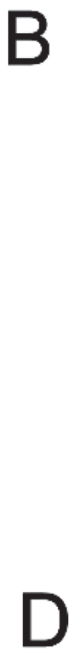
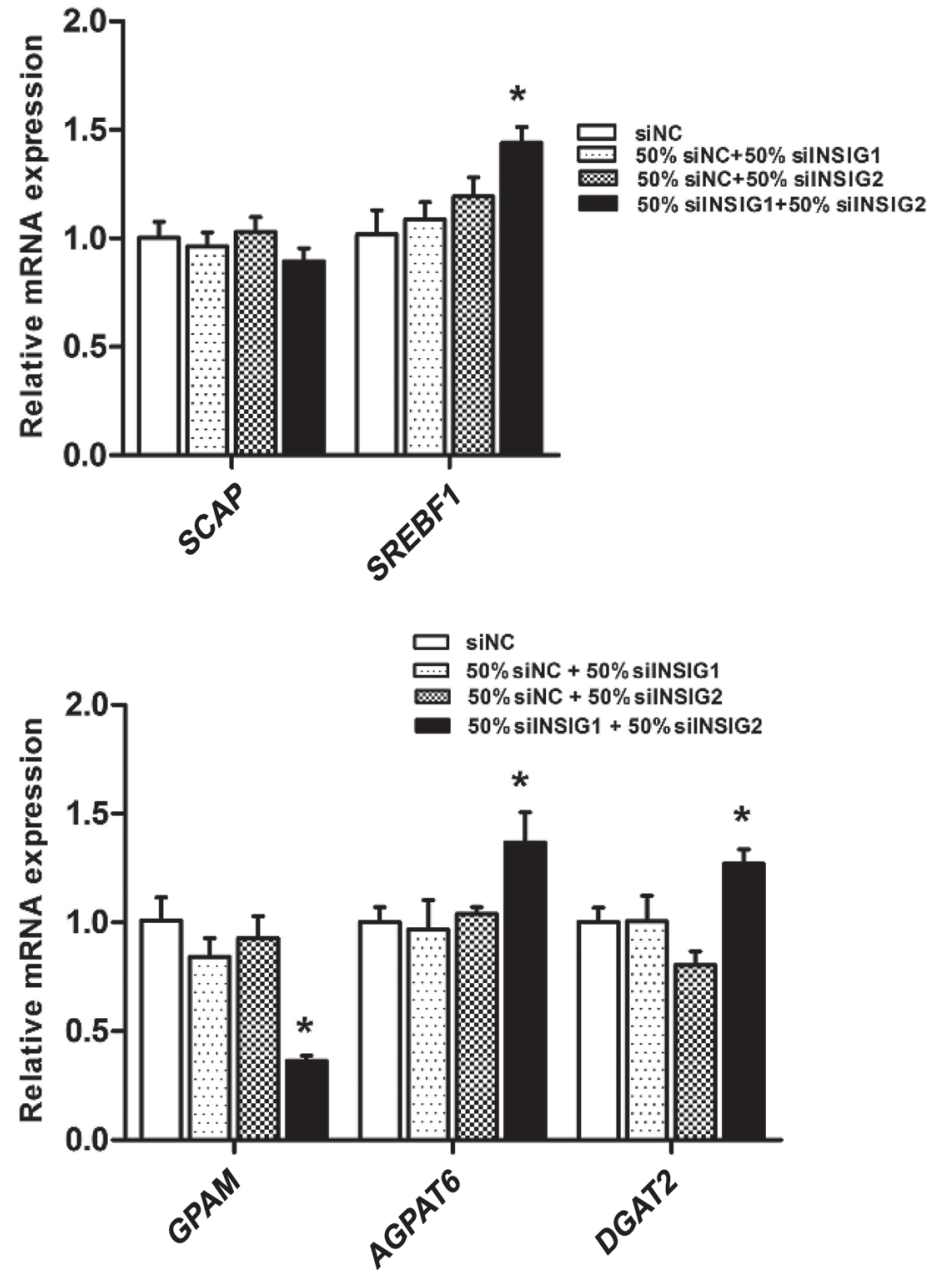

Figure 5. Simultaneous interference of INSIG1 and INSIG2 promotes mRNA expression of genes related to lipid synthesis in goat mammary epithelial cells (GMEC). The GMEC were incubated with small interfering RNA target INSIG1 (siINSIG1) or small interfering RNA target INSIG2 (siINSIG2) or a negative control (scrambled small interfering RNA; siNC) for 48 h and then collected with mRNA extraction. Interference of INSIG1 is presented as $50 \%$ siNC $+50 \%$ siINSIG1; interference of INSIG2 is presented as $50 \%$ siNC + 50\% siINSIG2; simultaneous interference of INSIG1 and INSIG2 is presented as 50\% siINSIG1 + 50\% siINSIG2. (A) The interference efficiency of INSIG1 and INSIG2. The mRNA expression levels of genes related to (B) transcription factors, (C) de novo fatty acid synthesis and desaturation, and (D) triacylglycerol synthesis in GMEC. Values are presented as means \pm SE from 3 individual cultures. $* P<0.05$ compared with control $($ siNC). 
adds an FA acyl-CoA to diacylglycerol to form TAG (Abeni et al., 2005; Bichi et al., 2013). The concentration of TAG in cells changes dynamically, with lipolysis also playing a role in terms of providing FA for mem- brane lipid synthesis and as sources of energy via oxidation. Both ATGL and HSL are key enzymes involved in the hydrolysis of TAG. The downregulation of GPAM and DGAT2 in response to overexpression of INSIG1
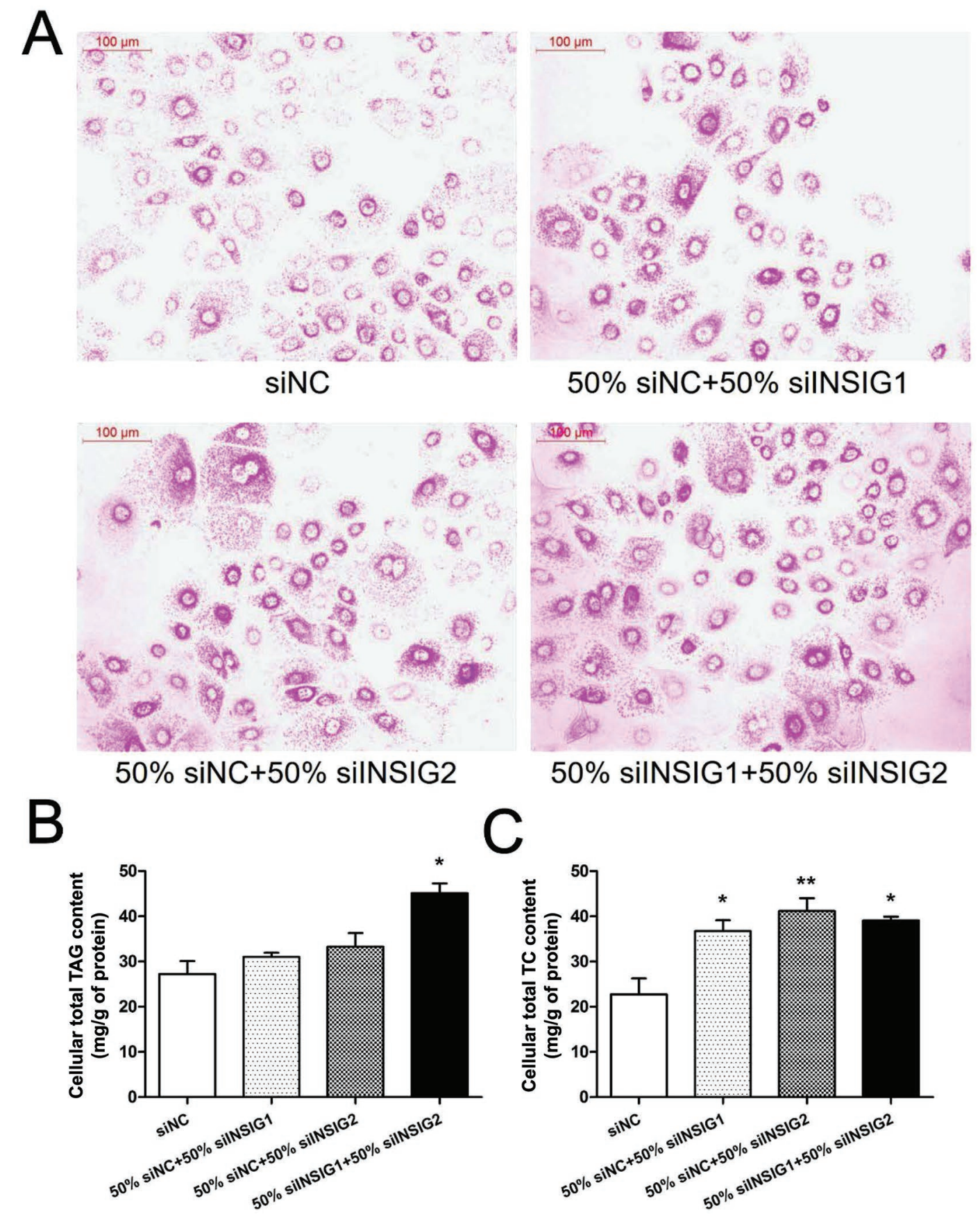

Figure 6. Simultaneous interference of INSIG1 and INSIG2 increased the accumulation of lipid droplet, concentration of cellular triacylglycerol (TAG), and total cholesterol (TC). The goat mammary epithelial cells (GMEC) were incubated with small interfering RNA target INSIG1 (siINSIG1) or small interfering RNA target INSIG2 (siINSIG2) or a negative control (scrambled small interfering RNA; siNC) and collected at $48 \mathrm{~h}$ for lipid droplet measurement and TAG and TC analysis. (A) Lipid droplet formation after Oil Red O treatment (scale bar: $100 \mu \mathrm{m}$ ). (B) The effect of interfering INSIG1 and INSIG2 on total TAG content in GMEC. (C) The effect of interfering INSIG1 and INSIG2 on total TC content in GMEC. Values are presented as means \pm SE from 3 individual cultures. The contents of TAG and TC are presented as milligrams per gram of protein. ${ }^{*} P<0.05$ and ${ }^{* *} P<0.01$ compared with control (siNC). 
indicated an inhibitory effect on TAG synthesis, also supported by the decrease in TAG content. This idea is further supported by previous data with mice in which overexpression of INSIG1 suppressed the transcription of genes related to TAG synthesis in liver (Engelking et al., 2004; Takaishi et al., 2004). Similar to lipogenesis, the data suggest that activation of INSIG1 also could decrease TAG synthesis.

The downregulation of AGPAT6 and DGAT2 coupled with a lack of change in GPAM in response to overexpression of INSIG2 reinforces the existence of complex mechanisms and regulatory pathways coordinating lipid metabolism (Loor et al., 2013). Although INSIG proteins decrease the activation of SREBF and the degradation of 3-hydroxy-3-methylglutaryl-coenzyme A reductase (HMGR) in nonruminants (Theesfeld and Hampton, 2013; Jiang et al., 2018; Johnson and DeBose-Boyd, 2018), other factors may regulate mRNA expression of GPAM. The decrease in expression of $H S L$ and $A T G L$ in response to INSIG upregulation may represent a self-protecting mechanism within mammary epithelial cells to control the intracellular concentration of lipid at a normal level.

The marked decrease in content of TAG and TC in response to overexpression of INSIG2 along with a modest decrease in content of TAG when INSIG1 was overexpressed suggested that TAG synthesis is mainly regulated by INSIG2, whereas TC synthesis is equally regulated by INSIG2 and INSIG1. This suggestion is supported by a previous study in which overexpression of INSIG2 compared with overexpression of INSIG1 significantly downregulated TAG content in murine plasma and liver (Takaishi et al., 2004). Furthermore, overexpression of INSIG2 resulted in a strong inhibition of adipogenic differentiation and lipid biosynthesis in adipose-derived stem cells (Chen et al., 2017).

The lack of change in mRNA expression of $S C A P$ and SREBF1 and TAG content in response to interference of INSIG1 agrees with results in murine plasma and liver (McFarlane et al., 2014), which indicated a compensatory effect of INSIG2 on lipid synthesis. The lack of difference in mRNA expression of genes related to lipid metabolism along with the lack of changes in content of TAG and accumulation of lipids when $I N$ SIG1 or INSIG2 were downregulated alone suggested that INSIG1 and INSIG2 proteins help maintain lipid homeostasis. Thus, we speculate that interference of one INSIG subtype may trigger a compensatory effect by the other subtype, resulting in no net changes in lipid synthesis. The significant increase in lipid accumulation in response to simultaneous interference of INSIG1 and INSIG2 agrees with previous studies in mice (McFarlane et al., 2014). The significant increases in total TAG and TC contents are consistent with a reported
4- to 6-fold increase in liver of hamsters (Engelking et al., 2005). The marked increase in content of TAG and TC and accumulation of lipids along with significant upregulation of SREBF1, ACACA, SCD1, AGPAT6, and DGAT2 indicated that INSIG1 and INSIG2 synergistically regulate lipid metabolism in GMEC.

\section{CONCLUSIONS}

The genes INSIG1 and INSIG2 induced a widespread suppression of TAG accumulation, lipolysis, and lipid droplet formation partly through controlling gene transcription in GMEC. Thus, INSIG1 and INSIG2 synergistically regulate various aspects of lipid metabolism in GMEC to control homeostasis. Understanding the regulatory role of INSIG in lipid metabolism in the lactating ruminant may contribute to the efforts in manipulating milk fat synthesis and secretion.

\section{ACKNOWLEDGMENTS}

This work was jointly supported by the National Natural Science Foundation of China (31702098, 31501983, 31772575), Young Talent Fund of University Association for Science and Technology in Shaanxi, China (20170202), National Post-Doctoral Science Foundation (2017M613230), and PhD research startup foundation of Northwest A\&F University (Yangling, Shaanxi, China; 2452016203).

\section{REFERENCES}

Abeni, F., L. Degano, F. Calza, R. Giangiacomo, and G. Pirlo. 2005. Milk quality and automatic milking: Fat globule size, natural creaming, and lipolysis. J. Dairy Sci. 88:3519-3529.

Bichi, E., G. Hervás, P. G. Toral, J. J. Loor, and P. Frutos. 2013. Milk fat depression induced by dietary marine algae in dairy ewes: Persistency of milk fatty acid composition and animal performance responses. J. Dairy Sci. 96:524-532. https://doi.org/10.3168/jds .2012-5875.

Bionaz, M., and J. J. Loor. 2007. Identification of reference genes for quantitative real-time PCR in the bovine mammary gland during the lactation cycle. Physiol. Genomics 29:312-319.

Bionaz, M., and J. J. Loor. 2008. Gene networks driving bovine milk fat synthesis during the lactation cycle. BMC Genomics 9:366.

Bionaz, M., B. J. Thering, and J. J. Loor. 2012. Fine metabolic regulation in ruminants via nutrient-gene interactions: saturated long-chain fatty acids increase expression of genes involved in lipid metabolism and immune response partly through PPAR- $\alpha$ activation. Br. J. Nutr. 107:179-191. https://doi.org/10.1017/ S0007114511002777.

Bonnet, M., L. Bernard, S. Bes, and C. Leroux. 2013. Selection of reference genes for quantitative real-time PCR normalisation in adipose tissue, muscle, liver and mammary gland from ruminants. Animal 7:1344-1353.

Chen, C. C., L. W. Hsu, K. T. Huang, S. Goto, C. L. Chen, and T. Nakano. 2017. Overexpression of Insig-2 inhibits atypical antipsychotic-induced adipogenic differentiation and lipid biosynthesis in adipose-derived stem cells. Sci. Rep. 7:10901.

Cheng, C., P. Ru, F. Geng, J. Liu, J. Y. Yoo, X. Wu, X. Cheng, V. Euthine, P. Hu, J. Y. Guo, E. Lefai, B. Kaur, A. Nohturfft, J. Ma, A. 
Chakravarti, and D. Guo. 2015. Glucose-mediated N-glycosylation of SCAP is essential for SREBP-1 activation and tumor growth. Cancer Cell 28:569-581.

Dong, X. Y., and S. Q. Tang. 2010. Insulin-induced gene: A new regulator in lipid metabolism. Peptides 31:2145-2150.

Engelking, L. J., H. Kuriyama, R. E. Hammer, J. D. Horton, M. S. Brown, J. L. Goldstein, and G. Liang. 2004. Overexpression of Insig-1 in the livers of transgenic mice inhibits SREBP processing and reduces insulin-stimulated lipogenesis. J. Clin. Invest. 113:1168-1175.

Engelking, L. J., G. Liang, R. E. Hammer, K. Takaishi, H. Kuriyama B. M. Evers, W. P. Li, J. D. Horton, J. L. Goldstein, and M. S. Brown. 2005. Schoenheimer effect explained - Feedback regulation of cholesterol synthesis in mice mediated by Insig proteins. J. Clin. Invest. 115:2489-2498.

Farr, V. C., K. Stelwagen, L. R. Cate, A. J. Molenaar, T. B. McFadden, and S. R. Davis. 1996. An improved method for the routine biopsy of bovine mammary tissue. J. Dairy Sci. 79:543-549.

Gong, Y., J. N. Lee, M. S. Brown, J. L. Goldstein, and J. Ye. 2006. Juxtamembranous aspartic acid in Insig-1 and Insig-2 is required for cholesterol homeostasis. Proc. Natl. Acad. Sci. USA 103:61546159.

Jiang, L. Y., W. Jiang, N. Tian, Y. N. Xiong, J. Liu, J. Wei, K. Y. $\mathrm{Wu}$, J. Luo, X. J. Shi, and B. L. Song. 2018. Ring finger protein 145 (RNF145) is a ubiquitin ligase for sterol-induced degradation of HMG-CoA reductase. J. Biol. Chem. 293:4047-4055.

Johnson, B. M., and R. A. DeBose-Boyd. 2018. Underlying mechanisms for sterol-induced ubiquitination and ER-associated degradation of HMG CoA reductase. Semin. Cell Dev. Biol. 81:121-128.

Kadegowda, A. K., M. Bionaz, L. S. Piperova, R. A. Erdman, and J. J. Loor. 2009. Peroxisome proliferator-activated receptor-gamma activation and long-chain fatty acids alter lipogenic gene networks in bovine mammary epithelial cells to various extents. J. Dairy Sci. 92:4276-4289.

Krapivner, S., E. Chernogubova, M. Ericsson, C. Ahlbeck-Glader, A. Hamsten, and F. M. van't Hooft. 2007. Human evidence for the involvement of insulin-induced gene 1 in the regulation of plasma glucose concentration. Diabetologia 50:94-102.

Krapivner, S., S. Popov, E. Chernogubova, M. L. Hellenius, R. M. Fisher, A. Hamsten, and F. M. van't Hooft. 2008. Insulin-induced gene 2 involvement in human adipocyte metabolism and body weight regulation. J. Clin. Endocrinol. Metab. 93:1995-2001.

Lee, J. H., H. S. Kang, H. Y. Park, Y. A. Moon, Y. N. Kang, B. C. Oh, D. K. Song, J. H. Bae, and S. S. Im. 2017. PPARalpha-dependent Insig2a overexpression inhibits SREBP-1c processing during fasting. Sci. Rep. 7:9958.

Lee, P. C., and R. A. DeBose-Boyd. 2010. Intramembrane glycine mediates multimerization of Insig-2, a requirement for sterol regulation in Chinese hamster ovary cells. J. Lipid Res. 51:192-201.

Li, J., K. Takaishi, W. Cook, S. K. McCorkle, and R. H. Unger. 2003. Insig-1 "brakes" lipogenesis in adipocytes and inhibits differentiation of preadipocytes. Proc. Natl. Acad. Sci. USA 100:9476-9481.

Lin, X. Z., J. Luo, L. P. Zhang, W. Wang, H. B. Shi, and J. J. Zhu. 2013. MiR-27a suppresses triglyceride accumulation and affects gene mRNA expression associated with fat metabolism in dairy goat mammary gland epithelial cells. Gene 521:15-23.

Linden, A. G., S. Li, H. Y. Choi, F. Fang, M. Fukasawa, K. Uyeda, R. E. Hammer, J. D. Horton, L. J. Engelking, and G. Liang. 2018. Interplay between ChREBP and SREBP-1c coordinates postprandial glycolysis and lipogenesis in livers of mice. J. Lipid Res. 59:475-487.

Loor, J. J., M. Bionaz, and J. K. Drackley. 2013. Systems physiology in dairy cattle: Nutritional genomics and beyond. Annu. Rev.
Anim. Biosci. 1:365-392. https://doi.org/10.1146/annurev-animal $-031412-103728$

Luo, J., Z. L. Deng, X. Luo, N. Tang, W. X. Song, J. Chen, K. A. Sharff, H. H. Luu, R. C. Haydon, K. W. Kinzler, B. Vogelstein, and T. C. He. 2007. A protocol for rapid generation of recombinant adenoviruses using the AdEasy system. Nat. Protoc. 2:1236-1247.

McFarlane, M. R., G. Liang, and L. J. Engelking. 2014. Insig proteins mediate feedback inhibition of cholesterol synthesis in the intestine. J. Biol. Chem. 289:2148-2156.

Mehlem, A., C. E. Hagberg, L. Muhl, U. Eriksson, and A. Falkevall. 2013. Imaging of neutral lipids by oil red $\mathrm{O}$ for analyzing the metabolic status in health and disease. Nat. Protoc. 8:1149-1154.

Oppi-Williams, C., J. K. Suagee, and B. A. Corl. 2013. Regulation of lipid synthesis by liver X receptor alpha and sterol regulatory element-binding protein 1 in mammary epithelial cells. J. Dairy Sci. 96:112-121.

Romero, T., M. C. Beltran, M. Rodriguez, A. M. De Olives, and M. P. Molina. 2013. Short communication: Goat colostrum quality: Litter size and lactation number effects. J. Dairy Sci. 96:7526-7531.

Shi, H. J. Luo, J. Zhu, J. Li, Y. Sun, X. Lin, L. Zhang, D. Yao, and H. Shi. 2013. PPAR gamma regulates genes involved in triacylglycerol synthesis and secretion in mammary gland epithelial cells of dairy goats. PPAR Res. 2013:310948.

Shi, H., H. Shi, J. Luo, W. Wang, A. B. Haile, H. Xu, and J. Li. 2014 Establishment and characterization of a dairy goat mammary epithelial cell line with human telomerase (hT-MECs). Nihon Chikusan Gakkaiho 85:735-743

Shi, H. B., C. H. Zhang, W. Zhao, J. Luo, and J. J. Loor. 2017. Peroxisome proliferator-activated receptor delta facilitates lipid secretion and catabolism of fatty acids in dairy goat mammary epithelial cells. J. Dairy Sci. 100:797-806.

Smith, E. M., Y. Zhang, T. M. Baye, S. Gawrieh, R. Cole, J. Blangero, M. A. Carless, J. E. Curran, T. D. Dyer, L. J. Abraham, E. K Moses, A. H. Kissebah, L. J. Martin, and M. Olivier. 2010. INSIG1 influences obesity-related hypertriglyceridemia in humans. J. Lipid Res. 51:701-708.

Takaishi, K., L. Duplomb, M. Y. Wang, J. Li, and R. H. Unger. 2004. Hepatic insig-1 or -2 overexpression reduces lipogenesis in obese Zucker diabetic fatty rats and in fasted/refed normal rats. Proc. Natl. Acad. Sci. USA 101:7106-7111.

Theesfeld, C. L., and R. Y. Hampton. 2013. Insulin-induced gene protein (INSIG)-dependent sterol regulation of $\mathrm{Hmg} 2$ endoplasmic reticulum-associated degradation (ERAD) in yeast. J. Biol. Chem. 288:8519-8530.

Wang, H., M. Zhao, N. Sud, P. Christian, J. Shen, Y. Song, A. Pashaj, K. Zhang, T. Carr, and Q. Su. 2016. Glucagon regulates hepatic lipid metabolism via cAMP and Insig-2 signaling: Implication for the pathogenesis of hypertriglyceridemia and hepatic steatosis. Sci. Rep. 6:32246.

Wang, Z., J. Luo, W. Wang, W. Zhao, and X. Lin. 2010. Characterization and culture of isolated primary dairy goat mammary gland epithelial cells. Sheng Wu Gong Cheng Xue Bao 26:1123-1127.

Yabe, D., R. Komuro, G. Liang, J. L. Goldstein, and M. S. Brown. 2003. Liver-specific mRNA for Insig-2 down-regulated by insulin: Implications for fatty acid synthesis. Proc. Natl. Acad. Sci. USA 100:3155-3160

Yang, T., P. J. Espenshade, M. E. Wright, D. Yabe, Y. Gong, R. Aebersold, J. L. Goldstein, and M. S. Brown. 2002. Crucial step in cholesterol homeostasis: Sterols promote binding of SCAP to INSIG-1, a membrane protein that facilitates retention of SREBPs in ER. Cell 110:489-500. 